\section{Dos sistemas de desinclusion de dientes retenidos: sistema resorte ballesta y sistema trampa de raton}

Two systems of desinclusion of retained teeth: system means crossbow and mouse trap system

\author{
Resumen \\ La presencia de un diente retenido es muy común en niños, excluyendo las terceras \\ molares son los mas frecuentemente impactados. Comúnmente la mayoría de estos \\ dientes son expuestos quirúrgicamente siguiendo su orientación ortodóntica dentro \\ de la arcada dental ${ }^{1}$. Dentro de las variaciones de tralamiento ortodóntico tenemos \\ el sistema "Ballista Spring" y el "Mouse Trap" que nos va a permitir solucionar \\ limitaciones de otros sistemas. 2,4,16
}

\section{Abstract}

The presence of retained teeth is very common in children, excluding third molars, they are the most frequently. Most of these tecth are exposed surgically following its orthodontic direction within the dental arches ${ }^{1}$. In orthodontic treatment we have the system "Ballista Spring" and the "Mouse Trap" that will allow us the solve limitations of another systems $2,4,16$

\section{Luciano Soldevilla Galarza'; Rolando Alarcón Olivera ${ }^{2}$ y Lorena Rodríguez Varas $^{3}$}

Departamento Académico de Estomatología Pediátrica, Facultad de Odontología, Universidad Nacional Mayor de San Marcos, Lima, Perú

2 Consulta privada, Av. La Molina 1013, Lima, Perú

${ }^{3}$ Consulta privada, Jr. Bolognesi 197 piso 2,Lima 33, Perú

E-mail; lsoldevillag@unmsm.edu.pe

Palabras clave: Canino incluido, tratamiento ortodóncico, resorte ballesta, trampa de ratón.

Key Words: Impacted canine, orthodontic treatment, ballista spring, mouse trap

\section{Introducción}

En un artículo anterior se hizo la revisión accrca de la incidencia, prevalencia, etiología, diagnóstico, localización radiográfica y tratamicnto-en el caso de piezas dentarias incluidas (caninos) ${ }^{1}$.

El predominio de los caninos incluidos por palatino se estima cerca de $85 \%$ de casos y por bucal solamente cerca del $15 \%$ de $\operatorname{casos}^{1-4}$.

Ericson y Kurol estiman que en $0.71 \%$ de niños en edad de 10 a 13 años, los incisivos permanentes tienden a reabsorberse debido a los caninos maxilares ectópicos. La reabsorción se puede encontrar desde los 10 años pero ocurre con frecuencia a $\operatorname{los} 8$ años ${ }^{3-5}$.

Los caninos maxilares tienen el período más largo del desarrollo, así como el curso más largo y más tortuoso y las coronas se relacionan de cerca con las raíces de las incisivos laterales ${ }^{4,5}$.

Hay muchos factores etiológicos posibles asociados a la impactación del canino, según las listas de Bishara las causas más comunes son resultado de una combinación de los siguientes factores. ${ }^{3,4,6-8}$
1. Las discrepancias en la longitud del arco.

2. Retención prolongada o pérdida temprana del canino deciduo.

3. Posición anormal del germen dentario.

4. La presencia de una hendidura alveolar.

5. Anquilosis.

6. Formación enquistada o neoplásica

7. Dilaceración de la raíz.

8. Origen iatrogénico.

9. Condiciones idiopáticas en ausencia de desarrollo evidente del incisivo lateral superior así como la variación de tamaño de la raíz y la sincronización de la formación de la raíz también han estado implicadas como factores.

Sin importar la etiología, los caninos maxilares impactados ocurren con bastante frecuencia ${ }^{2,3}$.

El diagnóstico del canino maxilar impactado se debe basar en exámenes clínicos y radiográficos ${ }^{1,3,4}$, fig. 1 .

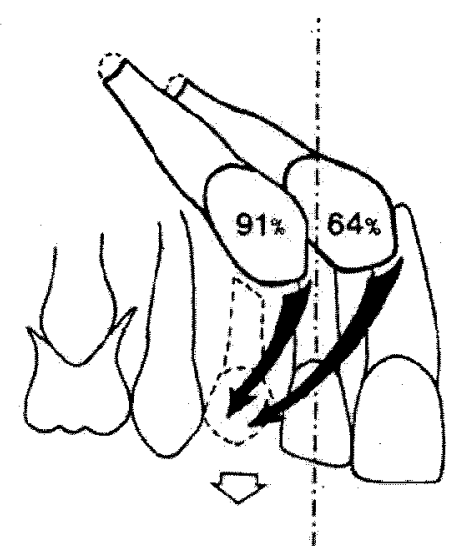

Fig. 1: Posición del canino en proceso de erupción.

La detección temprana y la prevención de la impactación por la extracción de piezas caducas disminuirían la necesidad del paciente de la cirugía oral y simplificarian el tratamiento ortodóntico ${ }^{2,3}$

La resorción de las raíces de los caninos se puede encontrar desde 10 años pero ocurre con más frecuencia a los 8 años.

Fl tratamiento de un canino maxilar impactado necesita ser integrado en el esquema ortodóntico total del tratamiento: 
1. Se debe preparar el espacio para el diente en el arco, que implica generalmente a los dientes adyacentes con o sin las extracciones.

2. Los otros dientes en el mismo arco $y$, a veces, en el arco antagonista deben proporcionar el anclaje para las fuerzas aplicadas al diente incluido para traerlo en la alineación.

3. La misma aparatología será utilizada para resolver la impactación y para tratar la maloclusión total, con solamente modificaciones de menor importancia ${ }^{9,10}$.

Cuando las medidas interceptivas fallan, la forma más común de trata: miento es la exposición quirúrgica y alineación ortodóntica, sin embargo, se debe tener en cuenta la salud a largo plazo de la gíngiva y la longitud inaceptable de la corona clínica considerada a menudo después de este procedimiento, actualmente se levanta una aleta mucoperiostica completa y un accesorio se enlaza a la superficie del diente sobre el cual se enlaza un alambre de ligadura, que debe emerger a través de la línea de la incisión, a través de la sutura ${ }^{4}$.

Dentro de las variaciones de tratamiento para la desinclusión de estas piezas tenemos el sistema "Ballista Spring ${ }^{\prime \prime 1,11}$,

Este sistema como la mayoría de tratamientos va a requerir de una intervención quirúrgica para la exposición de las coronas de las piezas a traccionar $^{7,11}$,

Con la mejora en los sistemas adhesivos se mejoró esta têcnica ya que anteriormente el diente impactado era. enlazado con un alambre de ligadura alrededor de la zona cervical al momento de la exposición quirúrgica, lo que requería canalizar y remover una porción amplia de hueso que cubre la corona del diente impactado dando lugar a una recuperación mas dolorosa por parte del paciente con respecto a la capacidad de comer, entre otras ${ }^{4, b, 12}$.

Se enlaza un alambre de ligadura al canino

para proporcionar un medio simple y flexible de tracción que asegure que el canino entre en erupción en una zona de la mucosa determinada ${ }^{4}$.

La dirección del tirón en la mayoría de los caninos ectópicos ubicados en el paladar y hacia la línea media primero debe ser retirando la corona canina lejos de las raíces de los incisovos centrales o laterales. Esta dirección distal inicial del tirón también requiere un componente vertical. Por el contrario, el canino impactado por bucal requerirá generalmente un tirón vertical con un componente distal pequeño. Para los caninos afectados tanto por palatino y bucal, la tracción necesita ser suave y continua y capaz de ser alterada en la dirección del canino cuando entre en erupción ${ }^{4}$.

\section{Sistema "Ballista Sping"}

Esta conformado por un alambre redondo de $0.14^{\prime \prime}, 0.16^{\prime \prime}$ o $0.18^{\prime \prime}$, los cuales acumulan energía al ser doblados sobre su eje longitudinal, este va a tener una sección horizontal que va a ingresar en un tubo doble colocado en el sistema de anclaje y en el slot de un bracket colocado en la primera premolar y una porción vertical que se levanta hacia la zona de la pieza retenida, fig. 2

Cuando la sección vertical es levantada hacia la pieza a traccionar se acumula energía en la sección horizontal y dicha sección vertical queda en forma de arco $^{11}$.

La fuerza del arco es proporcional a la longitud del alambre tanto en su porción vertical como horizontal. Un arco de $0.016^{\prime \prime}$ genera una fuerza de 60 a 100 grs. Un arco de $0.018^{\prime \prime}$ produce una fuerza de 120 a $150 \mathrm{grs}^{13}$.
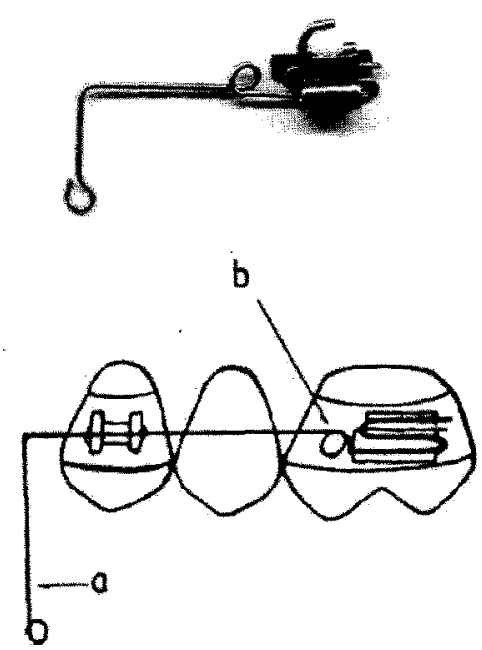

Fig. 2: Resorte tipo ballesta ligado al primer bicúspide y al molar, falta ligar la parte activa del resorte. (a)porción horizontal, (b) porción vertical.
El arco creado puede generar torsión de la molar para ello se confecciona un medio de anclaje que es un ATP, que va a incluir a la premolar también ya que se ha demostrado que esta puede sufrir rotaciones ya que a este nivel el arco actúa como un eje de bisagra ${ }^{11}$.

Para la confección del sistema de anclaje se adaptan bandas en las dos primeras molares y en las dos primeras premolares, se confecciona el ATP con alambre $0.045^{\prime \prime}$ y se ferulizan las bandas de las premolares, las bandas deben ser cementadas como una sola unidad y deben guardar paralelismo para lograr su inserción; es muy importante saber seleccionar los dientes de anclaje. Fiste anclaje puede ser utilizado en caninos impactados uni o bilateralmente ${ }^{11}$, fig. 3 .

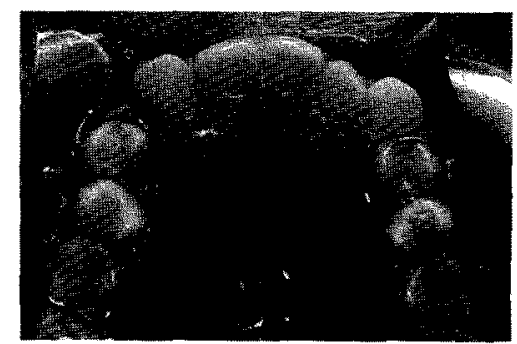

Fig. 3: Obsérvese el sistema de anclaje, un ATP de $0.045^{\prime \prime}$ soldado a cuatro bandas, dos en las primeras molares y dos en las primeras premolares fertizadas.

\section{Tratamiento de canino incluido}

Una vez ubicado el diente impactado clínica y radiográficamente se procede a la intervención quirúrgica en la que es expuesta una parte de la superficie del esmalte del diente impactado ${ }^{17}$.

Se lleva a cabo el acceso conveniente en el tejido blando (vestibular o palatino) para exponer el hueso que cubre la corona. Se tiene sumo cuidado para no tocar la raiz del canino retenido porque si se afecta el cemento aumentarían las posibilidades de anquílosis.

Si el desarrollo radicular todavía es incompleto, puede permitirse que el canino ya expuesto erupcione de manera pasiva.

Si el canino retenido presenta desarrollo completo de la raíz o se encuentra en posición precaria, se puede adherir un botón ortodóncico con resina autopolimerizable a la porción expuesta de su corona.

Se debe tener presente que es importante la conservación de la encia adherida que se da posterior a la retracción del diente impactado por vestibular, en el caso de piezas impactadas por 
palatino se debe dejar fibromucosa alrededor de este diente" ${ }^{n}$.

\section{Modificaciones del resorte en ballesta}

Si el resorte es doblado en sentido anterior $y$ posterior, se añade un componente en esa dirección, al vector vertical, fig. 4 .

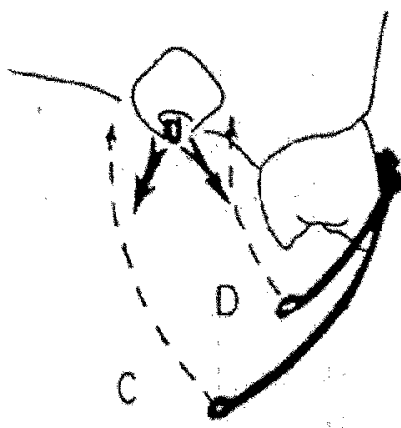

Fig. 4: Tracción vertical del canino

Si se alarga o se acorta, se añade una tracción lateral o media al canino, fig. 5.

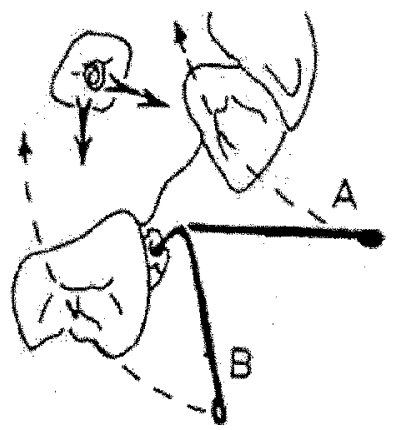

Fig. 5: Traccion lateral del canino

Si después de dos meses de tratamiento no se observa ningún progreso debido a lo severo de la impactación o a la resistencia de los tejidos puede aplicarse un segundo arco para incrementar la fuerza en el mismo tubo"

\section{Ventajas del Sistema}

- Permite el control de la erupción del diente al ejercer una fuerza en sentido vestibular para alejar la corona del canino de la raíz del lateral y evitar su reabsorción.

- Provee una fuerza continua, controlable y fácilmente modificable

- Disminuye al mínimo la molestia en el paciente y puede ser usado tanto en pacientes jóvenes como en adultos.
- Es de fácil manipulación.

- No se requiere de toda la aparatología fija.

- Duración del tratamiento de 6 a 12 meses en casos favorables ${ }^{31}$.

\section{Riesgos}

- Anquílosis del canino.

- Reabsorción apical de los dientes vecinos ${ }^{n}$.

\section{Conclusiones}

- Este sistema permite solucionar limitaciones que se tiene con los sistemas removibles, como la tracción de piezas impactadas por palatino.

- Disminuye la reabsorción radicular de los dientes adyacentes en comparación con los aparatos removibles ya que llevan el diente hacia vestibular para su posterior reposicionamiento en el arco, modificando asî su guía de erupción.

- Este tipo de resorte puede sufrir modificaciones para adaptarse a los requerimientos de cada paciente.

- No es necesaria la colaboración del paciente como en el caso de los aparatos removibles.

\section{Casos Clínicos (Figs. 6-18)}

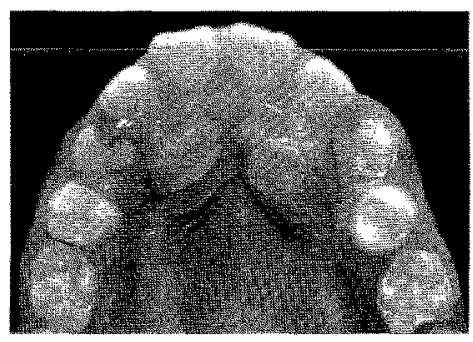

Fig. 6: Fotografía oclusal superior: obsérvese ausencia de piezas 13 y 23 ; piezas 14 y 24 en proceso de erupción

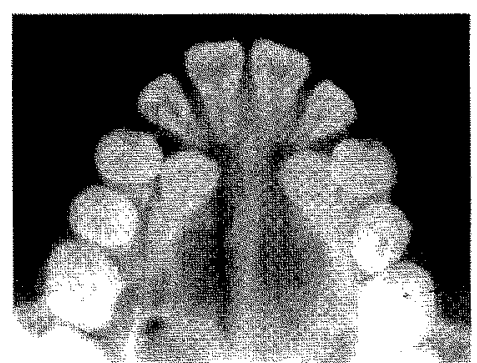

Fig. 7: Radiografía oclusal utilizada para localizar la ubicación de los caninos, los cuales se encuentran impactados por palatino.

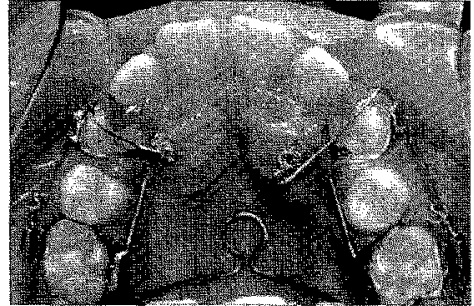

Fig. 8: Colocación del sistema de anclaje y arcos ballesta bilateralmente para la tracción de anbas piezas retenidas

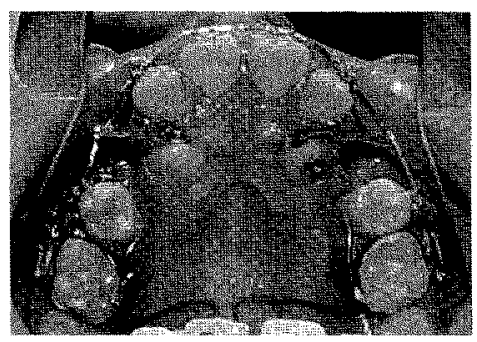

Fig. 9: Obsérvese la erupción de los carinos traccionados con los resortes lo suficiente para la colocación de brackets ya que se culminó con la colocación de aparatologia fija previa extraccion de primeras premolares para posicionar los caninos.

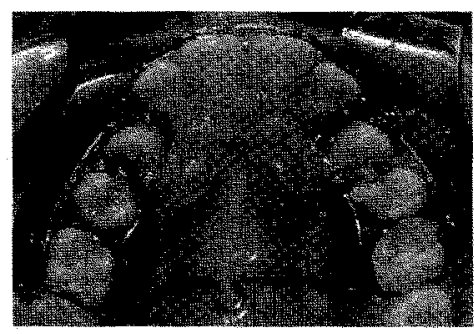

Fig. 10: Caninos posicionados ya en el arco y en proceso de erupción.

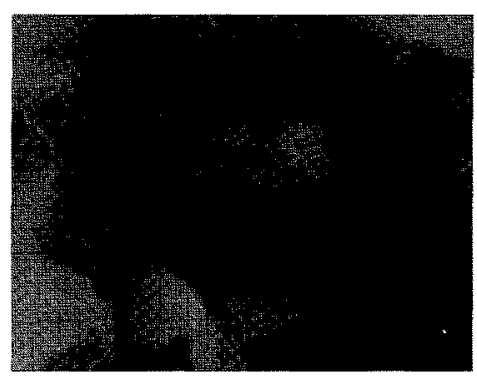

Fig. 11: Radiografías periapicales

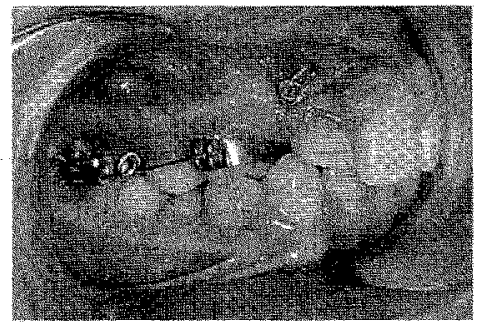

Fig.12: Canino orientado por vestibutar 


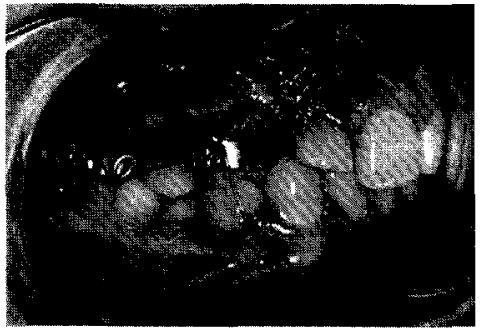

Fig. 13: Resorte pasivo

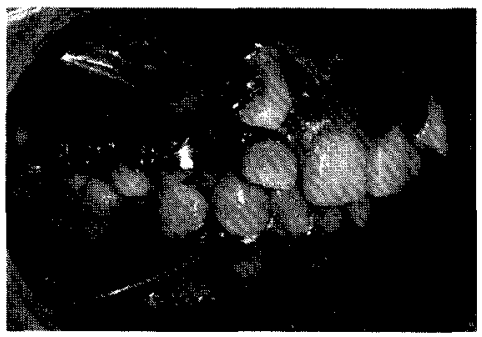

Fig. 14:- Resorte activo

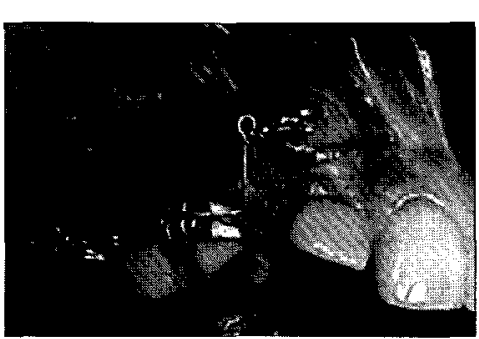

Fig. 15: Desinclusión parcial del canino

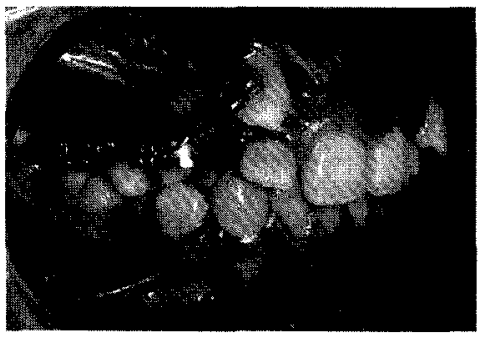

Fig. 16: Desinclusión total

\section{Sistema Trampa de Ratón}

El resorte se confecciona con alambre 0.014 " o $0.016^{\prime \prime}$ en acero inoxidable sobre un arco base rectangular como mínimo $0.017^{\prime \prime} \times 0.025^{\prime \prime}$. El àrco que sujeta al resorte es más seguro y conveniente si se coloca de primera molar a primera molar del lado opuesto.

El resorte "trampa de ratón" es un simple loop vertical con un pequeño helicoide en su extremo, el cual se encuentra orientado en sentido vertical al plano de oclusión y a nivel de la pieza a desincluir ${ }^{13}$.

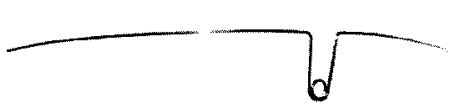

Fig. 17: Forma del resorte "trampa de ratón"

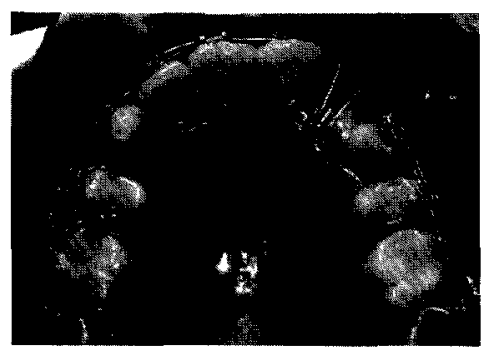

Fig. 18: Resorte activo

\section{Referencias}

1. Luciano Carlos Soldevilla Galarza, Oriel Orellana Manrique, Elizabeth Lorena Rodríguez Varas, Desinclusión de dientes retenidos mediante aparatologia removible Rev. Odontología Sanmarquina. 2003, 6 (12): 41- 45

2. John H. Warford Jr, Ram K. Grandhi, BDS, Dip Perio, Cert Ortho, Daniel E. Tira, Prediction of maxillary canine impaction using sectors and angular measurement American Journal of Orthodontics and Dentofacial Orthopedics. 2003, 124 (6).

3. Kuftinec MM, Shapira $Y$. The impacted maxillary canine: I. Review of concepts. ASDC Journal Dental Children. 1995,

\section{2(5):317-24.}

4. Harry S. Orton, M. Thérèse Garvey, Michael $\mathrm{H}$. Pearson, Extrusion of the ectopic maxillary canine using a lower removable appliance. 1995, 107 (4).

5. Angelo Moltola, Silvia Malerrari, "Abordaje quirúrgico y clasificación de las piezas dentarias incluidas", Journal de Clínica en Odontología. 1998/1999, 14 (6): 68-73

6. Yehoshua Shapira, Mladen M. Kuftine, Treatment of impacted Cuspids: The Hazard Lasso The Angle Orthodontis. 1981, 51 (3): 203 - 207.

7. Mariaca de Botero, L., Botero Mariaca P. "Una Técnica sencilla de distalización de caninos retenidos. Rev. de la Facultad de Odontología". Universidad de Antioquia, http: //chami.udea.edu. co/publicaciones/2002, 6-vol13-2-2002. html

8. Benjam in H. Williams, Diagnosis and Prevention of Maxillary Cuspid Impaction The AngleOrthodontist. 1981, 51 (1): $30-40$.

9. RG Oliver, JE Mannion and JM Robinson, Morphology of the maxillary lateral incisor in cases of unilateral impaction of the maxillary canine British Journal of Orthodontics. 1989, 16, 9-16

10. Charles A. Frank, Meridith Long, Jacksonville, Periodontal concerns associated with the orthodontic treatment of impacted teeth American Journal of Orthodontics and Dentofacial Orthopedics. 2002, $121(6)$

11. Jacoby Harry D. M. D. The "Ballista Spring". System for impacted teeth. American Journal of Orthodontics. 1979, 75 (2): 143-151.

12. S. Chaushu, A. Becker, R. Zeltser, N. Vasker and G. Ch aushu, Patients' perceptions of recovery after surgical exposure of impacted maxillary teeth treated with an open-eruptionsurgical-orthodontic technique. The European Journal of Orthodontics. 2004, 26(6):591-596.

13. Kornhauser, Abed, Harari, Becker The resolution of palatally impacted canines using palatal-ocelusal force from a buccal auxiliary American Journal of Orthodontics and Dentofacial Orthopedics. 1996, $110(5)$ 